\title{
Non-biting midges as hosts for water mite larvae in spring habitats in Luxembourg
}

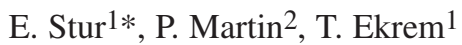 \\ ${ }^{1}$ Museum of Natural History and Archaeology, Norwegian University of Science and Technology, NO-7491 Trondheim, Norway. \\ 2 Christian-Albrechts-Universität, Zoologisches Institut (Department of Animal Ecology), Olshausenstr. 40, D-24098 Kiel, Germany.
}

\begin{abstract}
This study focuses on the host-parasite relationships between species of non-biting midges (Diptera: Chironomidae) and water mites (Acari: Hydrachnidia) in seven springs in Luxembourg. Emergence traps were operated through the season, and identification of the catches enabled an overview of species composition, abundance and host-parasite associations in the spring communities. Our results show that the Luxembourg springs have similar chironomid diversity to other springs in Europe, and that particular species are more often found in certain spring habitats. Furthermore, we show that prevalence of water mite parasitism is highly variable among the sampled chironomid hosts, and that there are several species which were not parasitized despite being numerous in our samples. The reasons for the latter observation are discussed, and most of the cases can be explained by general unavailability of host or parasite, or life cycle incompatibility.
\end{abstract}

Keywords : Chironomidae, Acari, abundance, diversity, parasitism.

\section{Introduction}

The family Chironomidae is frequently the most abundant and species rich insect group in freshwater springs (Gerecke et al. 1998, Lindegaard et al. 1998, Orendt 2000, Stur \& Wiedenbrug in press, Wagensonner 1992). Previous studies have recorded as many as 49 species in a single spring (Stur \& Wiedenbrug in press) and about 200 species are known to inhabit springs in Europe (Lindegaard 1995). Until now, only five species were known to be restricted to this particular habitat with certainty and rightly can be named crenobionts, whereas thirty seven European chironomids have been found mainly in springs and spring brooks and are thus classified as crenophilous (Lindegaard 1995). However, this study and a spring project in Berchtesgaden National Park have resulted in the discovery of eight possibly crenobiont species new to science (Stur \& Sæther 2004, Stur \& Ekrem in press,

\footnotetext{
* Corresponding author :

E-mail: Elisabeth.Stur@vm.ntnu.no
}

Stur \& Wiedenbrug 2005, unpublished data). Thus, the number of crenobiont chironomid species in Europe has more than doubled in the last few years. The chironomid fauna of European coldwater springs has previously been found to be dominated by members of the subfamily Orthocladiinae, followed by representatives of the tribe Tanytarsini within the subfamily Chironominae, and of the subfamily Diamesinae (Lindegaard 1995).

Springs are considered to be good sources for monitoring alterations in groundwater quality (Williams \& Williams 1998), and both chironomids and water mites are excellent reference organisms for groundwater biomonitoring due to their abundance and species richness in springs (Cantonati \& Ortler 1998, Lindegaard 1995, Orendt 2000). However, since water mites and chironomids interact closely with each other, and certain water mite species have the potential to be host specific (Efford 1963), it is useful to know more about their relationships and parasite-host associations for a more effective application of both groups as environmental indicators. 
Interactions between water mite larvae and their chironomid hosts have been investigated in lakes (e.g. Booth \& Learner 1978, Kouwets \& Davids 1984, Smukalla \& Meyer 1988, Stechmann 1980) and in more general terms (e.g. Hevers 1978, McLachlan 1999, McLachlan et al. 1999, Smith \& Oliver 1976, Wiles 1987), but rarely with relation to springs and spring brooks (e.g. Hevers 1978, McLachlan 1999, McLachlan et al. 1999, Smith \& Oliver 1976, Wiles 1987).

The study presented here is part of a larger project on the fauna of several springs in Luxembourg (see Gerecke et al. 2005). We give an overview of the parasitized and non-parasitized Chironomidae in the studied springs, and consider the taxonomic and numerical variation in water mite parasites on various chironomid hosts in the studied springs. We then discuss the possible explanations for the observed variations in host parasite associations.

\section{Material and methods}

Emergence traps were used to sample emerging insects and their attached water mite larvae in seven ground water springs in Luxembourg (traps were numbered E1-E7.). The $0.8 \mathrm{~m}$ by $0.8 \mathrm{~m}$ traps were placed over the springs or upper course of spring brooks, and operated from April to October 1999. Traps were emptied at intervals of two weeks. Fixative fluid was $75 \%$ ethyl alcohol. Gerecke, et al. (2005) give details of trap placement, spring typology, macroinvertebrates, and meiobenthos of the seven springs, including a faunistic list and comments on the biology of the water mites. Abiotic data on the here included springs are listed in Table 1. Qu11 is a rheohelocrene spring which consists of several small outlets in an open pasture. The spring itself is fenced, but livestock is held on the surrounding pasture in the summer months (Gerecke et al. 2005).
Thus, the spring water might be slightly enriched by cow-dung. Qu19 is a rheocrene spring situated in a young ash forest, and was probably used as a water source for a former nearby farm (Gerecke et al. 2005). Qu20 is a helocrene spring situated in a flat, rarely mowed meadow where willow-bushes give some shade to the spring (Gerecke et al. 2005). The spring dried out in late July, and there was no chironomid emergence from this site in August, September and October 1999. Qu21 is a rheohelocrene spring shaded by alder trees in the vicinity of a low oak forest which is neighboured by farm land. The spring mouths build a small marsh which is in part strongly disturbed by wild boars ( $\mathrm{Ge}-$ recke et al. 2005). The true spring mouth of the rheohelocrene Qu23 is dammed, and our emergence trap was placed 50 meters beneath the small dam. The area is a former forest which was cut and is now regenerating with birch, willow, broom, blackberry, raspberry etc. (Gerecke et al. 2005). Qu24 is a rheohelocrene spring part of a larger spring complex several outlets over a larger area. The ground is used as pasture and the spring complex is in part strongly disturbed $(\mathrm{Ge}-$ recke et al. 2005). Qu25 is a rheocrene spring in a beech forest. Calcareous deposits and tufa exist from about 20 meter below the spring mouth, and moss, small ash- and alder trees grow on the tufa (Gerecke et al. 2005). The E1 sample for the period 29 June $-13 \mathrm{Ju}-$ ly was lost.

Chironomids parasitized by mites were sorted out and placed in individually labelled vials. Attachment sites of the larval mites were noted before the larvae were removed from their hosts, fixed in Koenike's fluid and slide mounted in Hoyer's medium for identification under a light microscope. Attachment sites were determined by where the larval mouth parts were embedded. Detached (free) larvae, which were not associated with their hosts but present in samples, were

Table 1. Abiotic data from investigated springs (Gerecke et al. 2005).

\begin{tabular}{lllllll}
\hline Spring no. & Trap no. & $\begin{array}{l}\text { Altitude } \\
(\mathrm{m} \text { a.s.l. })\end{array}$ & Slope $\left(^{\circ}\right)$ & $\begin{array}{l}\text { Water flow } \\
(\mathrm{l} / \mathrm{s})\end{array}$ & $\begin{array}{l}\text { Temperature } \\
\left({ }^{\circ} \mathrm{C}, \text { April } 1999\right)\end{array}$ & $\begin{array}{l}\text { Conductivity } \\
(\mu \mathrm{S} / \mathrm{cm})\end{array}$ \\
\hline qu11 & E4 & 420 & 12.1 & 2 & $16.5^{*}$ & 200 \\
qu19 & E1 & 325 & 11.7 & 0.7 & 8.3 & 533 \\
qu20 & E2 & 500 & 2.4 & 1.5 & 7.9 & 116 \\
qu21 & E5 & 385 & 6.1 & 1 & 7.3 & 63 \\
qu23 & E3 & 450 & 4.8 & 1.5 & 7.4 & 207 \\
qu24 & E6 & 310 & 8.5 & 2 & 9.2 & 138 \\
qu25 & E7 & 265 & 10.6 & 1 & 8.4 & 621 \\
\hline
\end{tabular}

*Temperature measured in August 1998 
also counted and identified. Identification of the water mite larvae was enabled by associating larval stages with their adult counterparts through rearing. The adults were identified by using original descriptions and available revisions. The chironomid hosts were slide-mounted in Euparal and identified to genus level using Wiederholm (1989), and to species level using various revisions and original descriptions. As males could be identified to species, but most females could be identified to genus level only, we focused on the associations between water mite larvae and their male, chironomid hosts. Abundance of Chironomidae taxa refers to all males at a certain locality (both infected and non-infected individuals). Abundance of water mite larvae refers to total numbers of individuals of a species at a sample site. Each host specimen is registered with the same code as its parasite(s) to preserve the connection between host and parasite for future revisions. All parasite and host specimens are stored in the Musée National d'Histoire Naturelle Luxembourg (MHNL).

Prevalence was used as the number of individual hosts of a particular chironomid species infected by a parasite divided by the total number of examined hosts (Margolls et al. 1982). An adjusted prevalence was calculated by excluding from the calculation the hosts from springs where the parasites were not found. For instance, Krenopelopia sp. was only parasitized by Bandakia concreta in our samples, and $B$. concreta was found only in the springs E3 and E6. Krenopelopia specimens taken from spring E1, E2, E4, E5 and E7 were therefore omitted in the calculation of adjusted prevalence. In general prevalence, presence of host specimens at localities where the parasite is absent can give an incorrect impression of a parasites' success in parasiting a host species. This will not be the case in adjusted prevalence.

\section{Results}

\section{Diversity and abundance}

In total 7349 chironomid males were identified. Among these, 75 different chironomid species were recorded, including four species of Tanypodinae, 49 Orthocladiinae, and 22 Chironominae of which 15 species were Tanytarsini (Table 2). At least 27 of the recorded species have previously been recorded only from springs and spring-brooks, and can be regarded as crenophilous (Table 2). A total of 26 chironomid species and 391 male specimens were parasitized by water mite larvae $(35 \%$ of the species and $5 \%$ of the in- dividuals). In the two most abundant groups, Orthocladiinae and Tanytarsini, $26.5 \%$ and $66.7 \%$ of all species were affected respectively. In Trissopelopia longimana, Limnophyes pentaplastus and Polypedilum albicorne only one parasitized female was found.

The abundance of individual Chironomidae genera, species and specimens varied greatly among the seven studied springs (Table 2). For instance, species of Krenopelopia, Limnophyes, Paraphaenocladius, Rheocricotopus and Micropsectra were recorded in all springs, whereas Diplocladius, Krenosmittia, Smittia, Synorthocladius, Paracladopelma and Parapsectra species were detected in only one locality each. Twenty three species were found in one locality only, but two species, Limnophyes minimus and Krenopelopia sp., were recorded in all seven springs. Most of the site-specific species recorded were singletons or doubletons, and their absence from samples from the other springs could have been due to their general rareness. However, a few site specific species were found in large numbers at certain localities. Ninety five males of Krenosmitta boreoalpina, 19 males of Pseudorthocladius curtistylus, 20 of Thienemannia libanica, 22 of Micropsectra calcifontis and 69 of Neostempellina thienemanni were found only in E7. Heleniella ornaticollis, Tvetenia calvescens and Micropsectra attenuata were present only in E1 and E7 (5-156 specimens of each species per spring), and six Nanocladius parvulus and 88 Microtendipes tarsalis were found only in E5. Species diversity was relatively constant at each spring site (20-25 species), but in E3 we recorded a total of 38 species (nine singletons and doubletons). The average number of species in the Luxembourg springs was 25 , close to the average species number in European springs reported by Lindegaard (1995), while the number of specimens recorded for each species varied from one (e.g. Heterotrissocladius marcidus in E3) to almost 1100 (Stempellinella flavidula in E5), but mostly less than 50 individuals were recorded for each species (Table 2).

Sixteen species of water mites were found parasitizing male and female chironomids in the seven springs (Table 3). Thirteen of these (exceptions were Sperchonopsis verrucosa, Feltria rouxi and cf. Tiphys sp.) can be categorized as crenobionts and crenophiles (Gerecke et al. 2005). E1 was dominated by Sperchon thienemanni, Ljania bipapillata, Atractides fonticolus and Sperchon longissimus whereas E5 was dominated by Sperchon thienemanni, Ljania bipapillata and Lebertia sefvei. Most of the specimens recorded from E3 were Sperchon squamosus, Hygrobates norvegicus, Lebertia sefvei and Bandakia concreta. In E2, which 
Table 2. Recorded Chironomidae species from the seven springs (E1-E7) in Luxembourg. The letter $\mathrm{x}$ indicates number of specimens found, $\mathrm{x}=1-$

$10, x x=11-50, x x x>51$. Species which had water mite larvae attached are written in bold. Crenophilous species are highlighted in grey.

\begin{tabular}{|c|c|c|c|c|c|c|c|c|c|c|c|c|c|c|c|c|c|}
\hline$\partial 0^{2}$ & E1 & E 2 & E 3 & E 4 & E 5 & E 6 & E 7 & $\Sigma$ & & E1 & E 2 & E 3 & E 4 & E 5 & E 6 & E 7 & $\underline{\Sigma}$ \\
\hline TANYPODINAE & & & & & & & & & $\begin{array}{l}\text { Pseudorthocladius curtistylus } \\
\text { (Goetghebuer) }\end{array}$ & & & & & & & $\mathbf{x x}$ & 19 \\
\hline Krenopelopia sp. (spp.?) & $\mathbf{x x x}$ & $x$ & $\mathbf{x x x}$ & $x x$ & $x$ & $x x$ & $x$ & 248 & Pseudorthocladius filiformis (Kieffer) & & & $x$ & $x$ & & $x$ & & 20 \\
\hline Macrope/opia adaucta (Kieffer) & & $\mathbf{x x}$ & $x$ & $x$ & $x$ & $\mathbf{x x}$ & & 99 & $\begin{array}{l}\text { Pseudosmittia albipennis } \\
\text { (Goetghebuer) }\end{array}$ & & & $x$ & & $x$ & & & 3 \\
\hline Macropelopia notata (Meigen) & & & $\mathbf{x x}$ & $\mathbf{x}$ & & $\mathbf{x x}$ & & 44 & Rheocricotopus atripes (Kieffer) & $\mathbf{x}$ & $\mathbf{x x}$ & $\mathbf{x x}$ & $x$ & $x$ & $\mathbf{x x}$ & & 61 \\
\hline Trissopelopia longimana (Stæger) & & & $\mathbf{x}$ & & & & $\mathbf{x x}$ & 20 & Rheocricotopus effusus (Walker) & & $x$ & $\mathbf{x x x}$ & $x$ & $\mathbf{x}$ & $\mathbf{x}$ & & 208 \\
\hline ORTHOCLADIINAE & & & & & & & & & Rheocricotopus fuscipes (Kieffer) & & & & $x$ & $\mathbf{x x}$ & & & 20 \\
\hline Brillia bifida (Kieffer) & $\mathbf{x x}$ & $x$ & $x$ & $x$ & $\mathbf{x x}$ & & & 64 & Rheocricotopus sp. pr. godavarius & & & & & & & $\mathbf{x}$ & 3 \\
\hline Bryophaenocladius sp. 1 & $\mathbf{x}$ & & & & & & & 1 & $\begin{array}{l}\text { Smittia pr. contingens (Walker) sensu } \\
\text { Pinder }\end{array}$ & $\mathbf{x}$ & & & & & & & 1 \\
\hline Bryophaenocladius sp. 2 & & & & & & $x$ & & 1 & Synorthocladius semivirens (Kieffer) & & & & & & $\mathbf{x}$ & & 2 \\
\hline Chaetocladius dissipatus (Edwards) & & & $\mathbf{x}$ & & & & & 2 & Thienemannia fulvofasciata (Kieffer) & $\mathbf{x x x}$ & & $\mathbf{x}$ & & $\mathbf{x x}$ & & $x$ & 114 \\
\hline Chaetocladius laminatus Brundin & $\mathbf{x x x}$ & & $\mathbf{x x x}$ & & & & & 146 & Thienemannia gracilis (Kieffer) & $x$ & & & & & & & 1 \\
\hline Chaetocladius pr. piger (Goetghebuer) & & & $\mathbf{x x}$ & & & & & 11 & $\begin{array}{l}\text { Thienemannia libanica Laville \& } \\
\text { Moubayed }\end{array}$ & & & & & & & $\mathbf{x x}$ & 20 \\
\hline Corynoneura fittkaui Schlee & & & $\mathbf{x x}$ & & $\mathbf{x}$ & $\mathbf{x}$ & & 32 & Thienemanniella cf. obscura Brundin & & & $\mathbf{x}$ & & & & & 2 \\
\hline Corynoneura lobata Edwards & $\mathbf{x x}$ & $x$ & $\mathbf{x x}$ & $x$ & $\mathbf{x x x}$ & & $\mathbf{x x x}$ & 1200 & Tvetenia bavarica (Goetghebuer) & $\mathbf{x}$ & & & & & & & 4 \\
\hline Diplocladius cultriger Kieffer & & $x$ & & & & & & 8 & Tvetenia calvescens (Edwards) & $\mathbf{x x x}$ & & & & & & $\mathbf{x x}$ & 203 \\
\hline Eukiefferiella brevicalcar/tirolensis & & $\mathbf{x}$ & $\mathbf{x}$ & & $x$ & & & 4 & CHIRONOMINAE & & & & & & & & \\
\hline Gymnometriocnemus brumalis (Edwards) & & & $\mathrm{x}$ & & & & & 1 & CHIRONOMINI & & & & & & & & \\
\hline Heleniella ornaticollis (Edwards) & $\mathbf{x x}$ & & & & & & $\mathbf{x x}$ & 30 & Microtendipes tarsalis (Walker) & & & & & $\mathbf{x x x}$ & & & 88 \\
\hline Heleniella serratosioi Ringe & & & $\mathbf{x}$ & & & $\mathbf{x}$ & & 13 & Paracladopelma camptolabis (Kieffer) & & & & & & & $\mathbf{x}$ & 10 \\
\hline Heterotanytarsus apicalis (Kieffer) & & $\mathbf{x x}$ & $\mathbf{x x x}$ & & & $\mathbf{x x}$ & & 535 & Paratendipes subequalis (Malloch) & & & & $\mathbf{x}$ & & $\mathbf{x}$ & & 11 \\
\hline Heterotrissocladius marcidus (Walker) & & & $\mathbf{x}$ & & & & & 1 & Polypedilum (Pe.) tritum (Walker) & & & $\mathbf{x x}$ & & & & & 12 \\
\hline $\begin{array}{l}\text { Krenosmittia boreoalpina } \\
\text { (Goetghebuer) }\end{array}$ & & & & & & & $\mathbf{x x x}$ & 95 & Polypedilum ( $P$.) albicorne (Meigen) & & & & & $\mathbf{x x x}$ & & $x$ & 75 \\
\hline Limnophyes difficilis Brundin & & $\mathbf{x x}$ & $\mathbf{x x}$ & $\mathbf{x}$ & & $\mathbf{x}$ & & 36 & Polypedilum ( $T$.) scalaenum (Schrank) & & & $\mathbf{x}$ & $\mathbf{x x x}$ & & & & 78 \\
\hline Limnophyes habilis (Walker) & & $\mathbf{x}$ & & & & & & 1 & Polypedilum (U.) convictum (Walker) & $\mathbf{x}$ & & & & & & & 3 \\
\hline Limnophyes minimus (Meigen) & $x$ & $\mathbf{x x}$ & $\mathbf{x x}$ & $\mathrm{x}$ & $\mathbf{x}$ & $\mathbf{x}$ & $\mathbf{x}$ & 72 & TANYTARSINI & & & & & & & & \\
\hline Limnophyes natalensis (Kieffer) & & $x$ & $\mathbf{x x}$ & $\mathbf{x x}$ & $\mathbf{x}$ & $\mathbf{x x}$ & $x$ & 70 & Micropsectra attenuata Reiss & $x$ & & & & & & $x$ & 15 \\
\hline Limnophyes pentaplastus (Kieffer) & $\mathbf{x x}$ & & $\mathbf{x}$ & $x$ & $\mathbf{x x}$ & $x$ & $x$ & 72 & $\begin{array}{l}\text { Micropsectra calcifontis Stur \& } \\
\text { Ekrem }\end{array}$ & & & & & & & $\mathbf{x x}$ & 22 \\
\hline Metriocnemus albolineatus (Walker) & & & & $x$ & & $\mathbf{x}$ & & 5 & Micropsectra junci (Meigen) & & $\mathbf{x x}$ & $\mathbf{x x x}$ & $\mathbf{x x x}$ & $\mathbf{x x}$ & $\mathbf{x}$ & & 232 \\
\hline Metriocnemus caudigus Sæther & & $\mathbf{x x}$ & & & & & & 27 & Micropsectra logani Johannsen & & $\mathbf{x}$ & & & & & & 1 \\
\hline Metriocnemus eurynotus (Holmgren) & & & $\mathbf{x}$ & & & & & 6 & $\begin{array}{l}\text { Micropsectra longicrista Stur \& } \\
\text { Ekrem }\end{array}$ & & & & & $x$ & & $x$ & 8 \\
\hline Metriocnemus fuscipes (Meigen) & $\mathbf{x}$ & & & $\mathbf{x}$ & & & & 3 & Micropsectra notescens (Walker) & & & & & & & $x$ & 1 \\
\hline Nanocladius parvulus (Kieffer) & & & & & $\mathbf{x}$ & & & 6 & Micropsectra pallidula (Meigen) & & $\mathbf{x x x}$ & $\mathbf{x x}$ & $\mathbf{x}$ & & & & 108 \\
\hline Orthocladius (O.) oblidens (Walker) & $\mathbf{x x x}$ & & $x$ & & $\mathbf{x x x}$ & & $\mathbf{x}$ & 329 & Micropsectra robusta Stur \& Ekrem & & & $x$ & & & & & 1 \\
\hline Orthocladius (O.) rubicundus (Meigen) & & & & & & $\mathbf{x}$ & & $1 / \mathrm{E}$ & $\begin{array}{l}\text { Micropsectra schrankelae Stur \& } \\
\text { Ekrem }\end{array}$ & $\mathbf{x x x}$ & & & & & & $\mathbf{x x}$ & 172 \\
\hline Parakiefferiella gracillima (Kieffer) & & $\mathbf{x x x}$ & $\mathbf{x x x}$ & $\mathbf{x}$ & & & & 854 & Neostempellina thienemanni Reiss & & & & & & & $\mathbf{x x x}$ & 69 \\
\hline Parametriocnemus stylatus (Kieffer) & $\mathbf{x x x}$ & & $\mathbf{x x}$ & $x$ & $\mathbf{x x x}$ & $x$ & $\mathbf{x x}$ & 191 & Parapsectra nana (Meigen) & & & $\mathbf{x}$ & & & & & 2 \\
\hline Paraphaenocladius exagitans Strenzke & $\mathbf{x}$ & & & & & $\mathbf{x}$ & & 5 & Stempellina sp. & & & & $\mathbf{x x}$ & & & & 18 \\
\hline $\begin{array}{l}\text { Paraphaenocladius impensus s. str. } \\
\text { (Walker) }\end{array}$ & $x x$ & $x$ & $\mathbf{x x}$ & $\mathbf{x x}$ & $x$ & $\mathbf{x}$ & & 73 & $\begin{array}{l}\text { Stempellinella ciliaris } \\
\text { (Goetghebuer) }\end{array}$ & & & & & $x$ & & $x \mathbf{x x}$ & 37 \\
\hline Paraphaenocladius pseudirritus Strenzke & & & $\mathbf{x x x}$ & $\mathbf{x x}$ & $\mathbf{x x}$ & & $\mathbf{x}$ & 122 & Stempellinella flavidula (Edwards) & & & & & $\mathbf{x x x}$ & & $\mathbf{x}$ & 1109 \\
\hline \multirow[t]{2}{*}{$\begin{array}{l}\text { Pseudorthocladius cristagus Stur \& } \\
\text { Sæther }\end{array}$} & & $x$ & & & & & & 2 & $\begin{array}{l}\text { Tanytarsus heusdensis } \\
\text { Goetghebuer }\end{array}$ & & & $\mathbf{x x \mathbf { x }}$ & & $\mathbf{x x}$ & & & 154 \\
\hline & & & & & & & & & Total species & 22 & 20 & 38 & 24 & 25 & 22 & 25 & \\
\hline
\end{tabular}


Table 3. Number of water mite larvae found parasitizing male and female chironomids in the seven sample sites, $\mathrm{x}=1-10, \mathrm{x}=11-50$, $x x x>51$. The numbers include detached larvae.

\begin{tabular}{|c|c|c|c|c|c|c|c|c|}
\hline all mite larvae & E 1 & E 2 & E 3 & E 4 & E 5 & E 6 & E 7 & $\Sigma$ \\
\hline Atractides fonticolus (K. Viets) & $x x x$ & & & & & & $x x x$ & 203 \\
\hline Atractides pennatus (K. Viets) & & & & & & & $x$ & 6 \\
\hline Bandakia concreta Thor & & & $x x$ & & & $x$ & & 22 \\
\hline Feltria rouxi Walter & & & & & & $x$ & & 1 \\
\hline Hygrobates norvegicus (Thor) & & & $x x x$ & & & $x$ & & 89 \\
\hline Lebertia glabra Thor & & & & & & & $x x$ & 50 \\
\hline Lebertia holsatica K. Viets & $x$ & & & & & & & 2 \\
\hline Lebertia sefvei Walter & & & $x x x$ & & $x x$ & & & 101 \\
\hline Ljania bipapillata Thor & $x x x$ & & $x$ & & $x x x$ & & $x x$ & 526 \\
\hline Mideopsis willmanni (Viets) & & & & $x$ & & & & 1 \\
\hline Pseudofeltria scourfieldi Soar & & & & $x$ & & $x$ & & 5 \\
\hline Sperchonopsis verrucosa (Protz) & & & & & & & $x x$ & 22 \\
\hline Sperchon longissimus $\mathrm{K}$. Viets & $x x x$ & & & $x$ & & & $x$ & 122 \\
\hline Sperchon squamosus Kramer & & $x x x$ & $x x x$ & & $x$ & & & 152 \\
\hline Sperchon thienemanni Koenike & $x x x$ & & $x$ & $x$ & $x x x$ & & $x$ & 387 \\
\hline Pionidae (cf. Tiphys sp.) & & & & & & & $x$ & 1 \\
\hline$\Sigma$ & 655 & 58 & 286 & 12 & 503 & 7 & 169 & 1690 \\
\hline
\end{tabular}

dried out in the late summer and autumn, we recorded only one species (Sperchon squamosus). In the remaining springs, there was no particular species dominance, or the number of recorded specimens is low.

\section{Prevalence and emergence patterns}

The most abundant water mite species utilized more host species than did less common mites (Table 4). Some chironomid species were infested by more water mite larvae than others (Table 4). Of the male chironomids with more than 15 specimens examined, over half of Brillia bifida and Rheocricotopus fuscipes were parasitized, whereas $21-47 \%$ of Parametriocnemus stylatus, Micropsectra attenuata, Micropsectra schrankelae and Stempellinella ciliaris were infested. Corynoneura lobata, Heterotanytarsus apicalis, Orthocladius oblidens, Micropsectra pallidula, and Tanytarsus heusdensis had a prevalence $<9 \%$ despite being numerous in several springs. Males of Stempellinella flavidula carried few mites, but most specimens of this species $(99.1 \%)$ were recorded from one spring only. Thus, S. flavidula was available to fewer parasite species compared to hosts with a wider distribution.
A comparison of the emergence pattern of four common, parasitized chironomid species and their associated water mite larvae in E1 (Figure 1), shows that prevalence was comparatively high for the small catches of Brillia bifida, and that on average there were seven mite larvae on each chironomid (sampling period 20 April -18 May). Brillia bifida is parasitized by two water mite species in E1, Ljania bipapillata and Atractides fonticolus. The relative abundance of specimens of L. bipapillata compared to A. fonticolus was considerable higher in the spring than autumn, and the number of parasites per specimen was also lower late in the year.

The bivoltine Parametriocnemus stylatus was parasitized by the same mite species as Brillia bifida, but many of Sperchon thienemanni were also found attached to this species (Figure 1). In particular, this was true at the start of each emergence period when $41 \%$ and $53 \%$ of the attached mites were this species. Ljania bipapillata was found parasitizing only one other species in E1 (4 specimens of Orthocladius oblidens in the first emergence period), thus the high number of attached mites on B. bifida observed in April/May, was 
Table 4. Proportion of mite species on their male chironomid hosts. The numbers of infected chironomids ( $\left.0^{7} \sigma^{7}\right)$ can sporadically be higher than the total number of parasites for the single species due to mite detachment after sorting. Adjusted prevalence only includes chironomids from sample sites where suited mite parasites where found (see table 3 and text for further explanation).

\begin{tabular}{|c|c|c|c|c|c|c|c|c|c|c|c|c|c|c|c|c|c|c|c|}
\hline $\begin{array}{l}\% \text { mite species on } \\
\text { chironomid host }\end{array}$ & 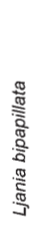 & 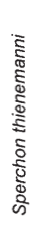 & 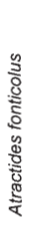 & 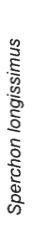 & 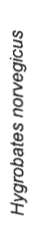 & 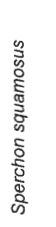 & 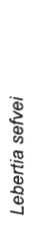 & 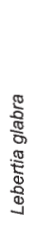 & 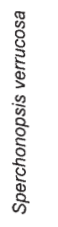 & 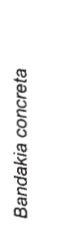 & 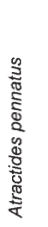 & 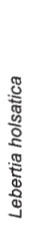 & 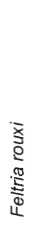 & $\begin{array}{l}\frac{0}{0} \\
\frac{\pi}{0} \\
\frac{0}{0} \\
\frac{0}{2}\end{array}$ & 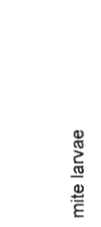 & 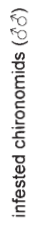 & 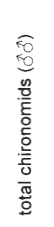 & 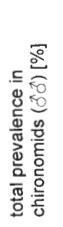 & 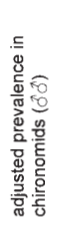 \\
\hline Krenopelopia spp. & & & & & & & & & & 66.7 & & & & & 6 & 7 & 248 & 3 & 7 \\
\hline Brillia bifida & 46.5 & & 20.8 & & 5.7 & & & & & & & & & & 160 & 34 & 64 & 53 & 56 \\
\hline Corynoneura lobata & & 5.0 & 21.9 & & & & & & 69.2 & & & & & & 38 & 37 & 1200 & 3 & 3 \\
\hline Heleniella ornaticollis & & & 1.0 & & & & & & & & & & & & 1 & 1 & 30 & 3 & 3 \\
\hline Heterotanytarsus apicalis & & & & & 2.9 & & & & & & & & & & 1 & 1 & 535 & $<1$ & $<1$ \\
\hline Orthocladius oblidens & 6.4 & & 3.1 & & & & & & & & & & & & 22 & 15 & 329 & 5 & 5 \\
\hline Orthocladius rubicundus & & & & & & & & & & & & & 100 & & 1 & 1 & 1 & 100 & 100 \\
\hline Parametricnemus stylatus & 16.5 & 13.8 & 10.4 & & & & & 4.3 & & & & 50.0 & & & 83 & 41 & 191 & 21 & 22 \\
\hline Rheocricotopus atripes & 2.7 & & & & 2.9 & & & & & & & & & & 9 & 2 & 61 & 3 & 4 \\
\hline Rheocricotopus effusus & 2.4 & & & & 60.0 & & & & & & & & & & 28 & 20 & 208 & 10 & 10 \\
\hline Rheocricotopus fuscipes & 6.7 & & & & & & & & & & & & & & 20 & 11 & 20 & 55 & 58 \\
\hline Tvetenia bavarica & & 1.9 & & & & & & & & & & & & & 3 & 1 & 4 & 25 & 25 \\
\hline TVetenia calvescens & & 10.0 & & 4.7 & & & & & & & 100 & & & & 23 & 24 & 203 & 12 & 12 \\
\hline Microtendipes tarsalis & 1.0 & 1.3 & & & & & & & & & & & & & 5 & 4 & 88 & 5 & 5 \\
\hline Micropsectra attenuata & 0.3 & 1.9 & 3.1 & 2.3 & & & & & & & & & & & 8 & 7 & 15 & 47 & 47 \\
\hline Micropsectra calcifontis & & & 1.0 & & & & & & & & & & & & 1 & 1 & 22 & 5 & 5 \\
\hline Micropsectra junci & 0.7 & 6.9 & & & 14.3 & 31.3 & 58.3 & & & & & & & & 42 & 27 & 232 & 12 & 12 \\
\hline Micropsectra longicrista & 0.3 & 2.5 & & & & & 12.5 & & & & & & & & 8 & 3 & 8 & 38 & 38 \\
\hline Micropsectra notescens & & & & & & & & & & & & & & 100 & 1 & 1 & 1 & 100 & 100 \\
\hline Micropsectra pallidula & & & & & & 15.6 & 8.3 & & & & & & & & 7 & 9 & 108 & 8 & 9 \\
\hline Micropsectra schrankelae & 0.3 & 12.5 & 11.5 & 58.1 & & & & 95.7 & & & & & & & 79 & 48 & 172 & 28 & 28 \\
\hline Stempellinella ciliaris & & & 9.4 & & & & & & & & & & & & 9 & 9 & 37 & 24 & 33 \\
\hline Stempellinella flavidula & 15.8 & 23.1 & 7.3 & & & & & & & & & & & & 91 & 81 & 1109 & 7 & 7 \\
\hline Tanytarsus heusdensis & & 0.6 & & & 11.4 & 3.1 & & & & & & & & & 6 & 6 & 154 & 4 & 4 \\
\hline detached mite larvae & 0.3 & 20.4 & 10.4 & 34.9 & 2.9 & 50.0 & 20.8 & & 30.8 & 33.3 & & 50.0 & & & 89 & & & & \\
\hline total number of mites & 297 & 160 & 96 & 43 & 35 & 32 & 24 & 23 & 13 & 9 & 5 & 2 & 1 & 1 & 741 & 391 & 5040 & & \\
\hline
\end{tabular}

probably influenced by the comparatively low number of Parametriocnemus stylatus available as hosts in the same period.

The univoltine Micropsectra schrankelae had peak emergence in mid-May to mid-July, the period when both $B$. bifida and $P$. stylatus were absent, or in low numbers in our E1 trap. Two mite species, $S$. thienemanni and A. fonticolus, switched hosts during this period, and become abundant on Micropsectra schrankelae (Figure 1). This chironomid also carried a third species, Sperchon longissimus. Except for one recorded larvae on Micropsectra attenuata, Micropsectra schrankelae was the only host for S. longissimus in E1. However, this mite has been recorded in small numbers on Tvetenia calvescens in other springs.

Males of T. calvescens have low prevalence in E1, and infestation was most noticeable in the weeks of high emergence. Except for two specimens of $S$. longissimus on one $T$. calvescens male, $S$. thienemanni was the dominant parasite on $T$. calvescens. This was also true for females of this species, 37 of which carried 58 specimens of $S$. thienemanni.

\section{Discussion}

\section{Diversity and abundance}

Considerable Chironomidae species diversity and variation between the sites was recorded in the seven Luxemburg springs. In a comparable study of three ground water springs in the German Alps, Stur \& Wiedenbrug (in press, http://www.nationalpark-berchtesgaden.de/html/chironomiden.html) recorded 68 chironomid species, 30 of which also are found in the investigated Luxembourg springs. Although there were quite a few species in common, more than half of the species were in our comparison only found at the Luxembourg sites. As has been found in other studies 


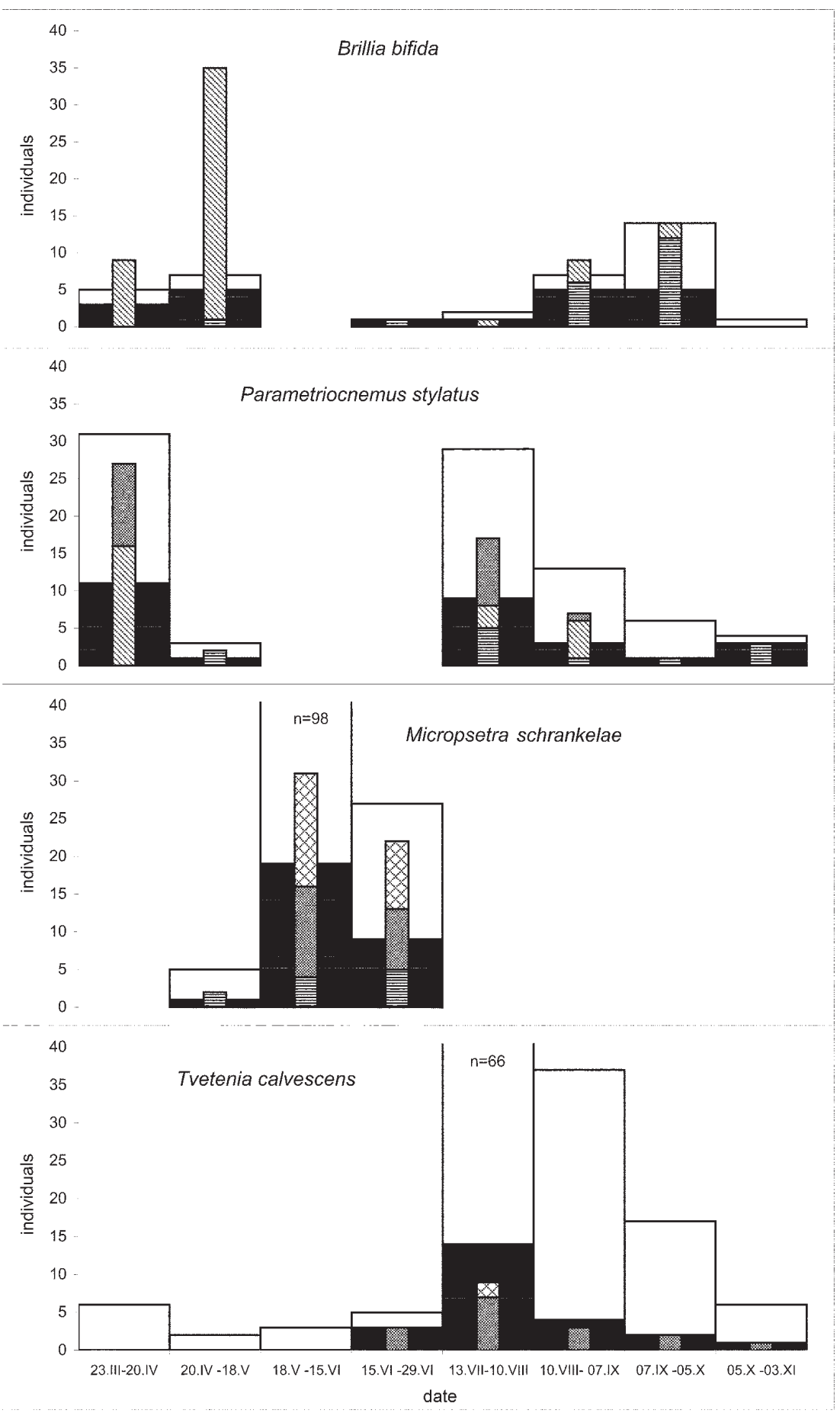

Fig. 1. Abundance of four Chironomidae species and their water mite parasites in a Luxembourg spring (E1) in 1999. Thick bars : $\square=$ non parasitized, $\mathbf{\square}=$ parasitized Chironomidae. Thin bars : III = Ljania bipapillata, $==$ Atractides fonticolus, $\Delta \diamond=$ Sperchon longissimus, $\square=$ Sperchon thienemanni. Sample from June 29 to July 13 was lost and could not be evaluated. 
in Germany and Denmark (e.g. Gümbel 1976, Lehmann 1971, Lindegaard 1995, Nolte 1991, Stur \& Wiedenbrug in press) species of the subfamily Orthocladiinae dominated both chironomid species richness and abundance. In our samples, $65.3 \%$ of the species and $64.1 \%$ of the male specimens were orthoclads. We consider males to provide good measures of species abundance and diversity, although they were slightly less abundant than females in our samples $(46 \%$ males). Our data on Orthocladiinae dominance are considerably lower than the $75-86 \%$ species dominance and 90-97\% abundance dominance Gümbel (1976) recorded in two springs in Hessen (Germany). However, Gümbel's sampling method differs from ours in several aspects, thus the numbers are not directly comparable. The chironomid communities of three investigated springs in the German Alps (Stur \& Wiedenbrug in press) on the other hand were sampled using the same methodology as in the present study. In these springs, the orthoclad species dominance $(69 \%)$ was close to that observed in the Luxembourg springs. In contrast, the North American spring fauna has been estimated to include only $42 \%$ orthoclads, but about $21 \%$ of species in the tribe Chironomini (Ferrington 1998). The larger percentage of Chironomini in North American springs is in strong contrast to the $6 \%$ recorded in European coldwater springs (Lindegaard 1995, this study), and may be explained by greater water temperature and nutrient gradients in the investigated North American springs (Ferrington 1998). Differences in species composition, richness and abundance can be explained by differences in abiotic factors such as water flow, temperature and alkalinity, and variation in microhabitats also influences species composition in springs (Lindegaard 1995). The reasons for the observed site specificity and variations in species abundance were not the focal points of this study, but it is expected that spring localities with similar abiotic characterisation also show similarities in species composition. A discussion on how some of these factors influence species specificity and richness is given by Lindegaard (1995). The most important parameters influencing the mite fauna in the studied springs have been shown to be the substrate composition, the water chemistry and shading of the springs by surrounding vegetation (Gerecke et al. 2005).

\section{Prevalence}

Unless specific water mite species detach more easily from certain chironomid species than others, infestation data on chironomid hosts are should be comparable for each mite species. However, the proportion of detached water mite larvae varied between mite spe- cies (Table 4), and was related to the species' preferred attachment sites. With the exception of Lebertia glabra, all mite species which were attached mainly to the host's thorax had a significantly higher proportion of detached larvae (Martin \& Stur 2005). Thus, one must include detached larva when quantitatively comparing the different water mites' success in finding and attaching to a host.

The prevalence of the Chironomidae in E1, E5 and E7 is higher than at the other sites although the number of individuals and species is similar. This can probably best be explained by the presence of the three most generalist species Ljana bipapillata, Sphercon thienemanni and Atracides fonticolus in high numbers at these sites (Table $3 \& 4$ ).

Interestingly, several chironomid species were not found to be parasitized by water mite larvae despite being numerous at several localities (Table 2). Apparently these species for some reason must be unfit as hosts for the water mite species they coexist with. Actually, quite few of these chironomid species have been recorded as host for water mite larvae, although Polypedilum convictum, a rare species in our samples, have been found to be quite heavily parasitized by stream living water mites (Martin \& Otto 2004). Several water mite species obviously accept more than one chironomid species as host (Table 4). Thus, host specificity can hardly alone be used to explain the lack of parasites on abundant chironomid species. Are certain chironomid species parasite-free because they are unattractive to the available mites, like Smith B.P. \& McIver (1983) found for some Aedes species and their potential Arrenurus parasites? Or, is it due to life cycle incompatibilities and general unavailability? The answers might be different for different host species.

The two Chaetocladius species with high abundance both occur in October and November, but both Lehmann (1971) and Lindegaard et al. (1975) report Chaetocladius laminatus to be very abundant in helocrenes in early spring (February to April/May). Parakiefferiella gracillima was found only in early spring. It is possible that we just missed the spring peak for $C$. laminatus in our samples, but the late (and early) emergence could make the Chaetocladius species and $P$. gracillima unavailable as hosts for many water mite species, since water mites that utilize holometabolous hosts typically mate in autumn and the females remain in the obligatory reproductive diapause until the following spring (Smith I.M. et al. 2001). One would therefore expect the prevalence to be low in the early spring as most species have not yet developed larvae, and low towards the end of the season as most mites prepare for 


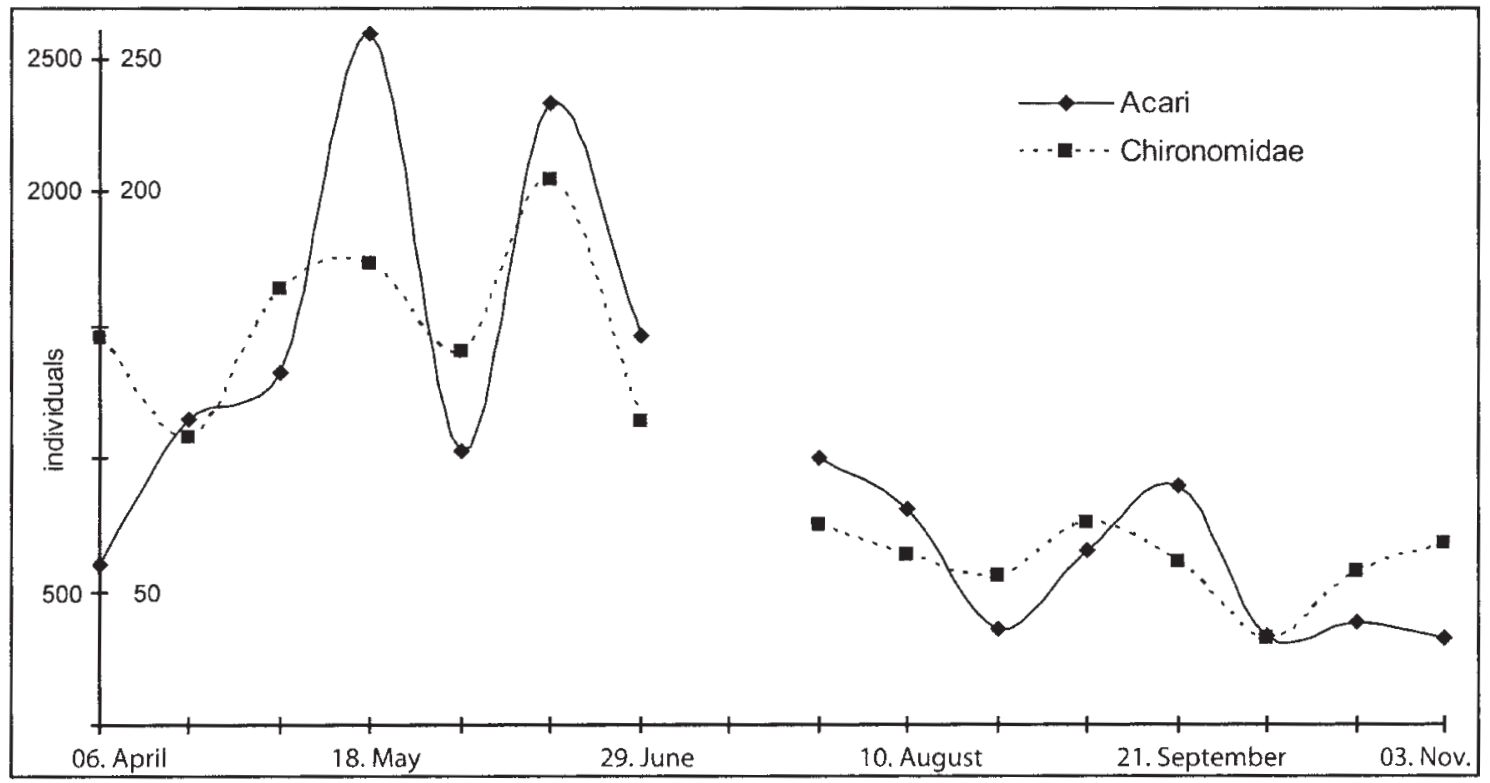

Fig. 2. Total frequency of chironomids (male and female) and water mite larvae in the emergence traps at seven Luxembourg springs between April 6 and November 3, 1999. Units on y-axis are 500 individuals for chironomids and 50 individuals for mites. The broken line indicates missing sample from E1 in the period between June 29 and July 13.

diapause in the adult stage. This pattern is present in our data (Figure 2, April and November). Nolte (1991) also recorded two species of Chaetocladius in mosses, and a moss-dwelling life style might be an additional reason for the lack of observed water mite parasites in our sampled Chaetocladius.

Corynoneura fittkaui, apparently escaped infestation at all sites where it was present, although Corynoneura lobata was parasitized by four different water mite species, Sperchonopsis verrucosa, Sperchon thienemanni, Atractides fonticolus and once by Ljania bipapillata. All these mite species were absent or in small numbers at E3 where more than ten Corynoneura fittkaui were collected. There seem to have been only small populations of $C$. fittkaui in E5 and E6. Thus, it appears that the potential and perhaps most probable parasites of $C$. fittkaui would have little chance to find this species in our springs.

The two Macropelopia species were found in large numbers, but only in the helocrene and rheohelocrene springs (E2-E6, Table 2). Lindegaard et al. (1975) also found relatively large numbers of Macropelopia notata in the moss carpet of the Ravnkilde spring in Denmark, and Lehmann (1971) reported both species from a section of the river Fulda which is lined with helocrene springs. Fittkau (1962) noted that M. notata and
M. adaucta are cold stenotherms and krenobiont and found in moss-living communities in soft water, helocrene springs. Thus, we believe that the habitat preference of the two species is moss, and that the semi-terrestrial lifestyle in the Luxembourg springs makes them less available to aquatic water mite larvae.

Four out of five of our recorded Limnophyes and all Metriocnemus, Paraphaenocladius, Pseudorthocladius, Pseudosmittia and Thienemannia species had no attached water mite larvae. Only one terrestrial mite larva was found on one specimen of Limnophyes pentaplastus. The well documented terrestrial and semiterrestrial life style of many species in these genera (Cranston et al. 1989, Lindegaard 1995, Nolte 1991, Sæther 1990, Strenzke 1950, Thienemann 1954) is possibly the reason why we found no water mite larvae utilizing them as hosts. However, this cannot be the whole story since moss-dwelling species like Tvetenia calvescens and T. bavarica were parasitized in the two springs where they occur. Except for Thienemannia fulvofasciata, Paraphaenocladius pseudirritus and Chaetocladius laminatus, all the non-parasitized moss-dwelling species discussed above were absent or in very few numbers in E1, E5 and E7. This means that most moss-dwelling chironomid species seldom would encounter larvae of Sperchon thienemanni and Atrac- 
tides fonticolus, two of the most numerous and probably generalist mite species in these springs (Table 3 and 4). Thienemannia fulvofasciata, Chaetocladius laminatus and Krenosmittia boreoalpina do not fit this picture, and at least $T$. fulvofasciata and $K$. boreoalpina need so far unmentioned explanations for not being utilized as water mite hosts. At least three explanations appear probable: either there are no water mite species parasitizing these potential hosts, the water mite species which could utilize the hosts are absent from the actual springs, and/or the host species are efficient in avoiding infestation by the water mites present. The two latter explanations seem probable for Krenosmittia boreoalpina because male and females of this species were parasitized by at least three different water mite species in two different alpine springs in the Nationalpark Berchtesgaden, Germany (unpublished data). There are to our knowledge no recorded water mite parasites of Thienemannia fulvofasciata. There were indications of parasitation on Thienemannia libanica by Sperchon sp. in the Berchtesgaden springs, but this could not be observed in E7, the only investigated spring emergence in Luxembourg with $T$. libanica. However, the three Sperchon species recorded in the Luxembourg springs (Table 3) were absent or only found once in E7, although two of them (S. thieneman$n i$ and S. longissimus) were, like Thienemannia fulvofasciata, numerous in E1. Thus, apparently T. fulvofasciata is efficient in avoiding the Sperchon generalists, or at least the Sperchon species found in E1 prefer other hosts (Table 4).

Within the subfamily Chironominae, three abundant species were never parasitized by water mite larvae in our study: Polypedilum (Pentapedilum) tritum, Polypedilum (Tripodura) scalaneum and Neostempellina thienemanni. The two Polypedilum species are also found in habitats other than springs, and although we were not able to find any documented proof of water mite - chironomid associations in the literature, it is reasonable to believe that mite parasites occur on these species in other habitats since several Polypedilum species frequently are used as hosts (Martin 2004, Martin \& Otto 2004, Martin et al. 2002, Smith I. M. \& Oliver 1976, 1986, Ullrich 1978, this study). The larvae of the tanytarsine Neostempellina thienemanni are moss-dwelling and have been found exclusively in alkaline springs and streams strongly influenced by limestone (Reiss 1984). The larvae build strong, transportable, calcareous cases (Reiss 1984, own observation) in which the pupae resides until emergence. The habitat preference of the larvae of this species and perhaps the tight cases limit its availability as a host
(Table 3), but other than that we have no apparent explanation to why $N$. thienemanni was not parasitized.

Is there any reason to believe that certain chironomid species have early or late-season emergence to avoid parasitism by water mite larvae? Our data are probably insufficient to indicate this, but none of the parasitized chironomids in our springs had a very early or late emergence, yet three unparasitized species did. Lanciani \& Boyt (1977) found reduced fecundity in miteparasitized females of Anopheles crucians (Culicidae), and negative effects on host survival and host population size have been recorded also for members of other insect orders (Smith B. P. 1988, Smith I.M. et al. 2001). If the same is true for mite infested Chironomidae, parasite avoidance by late or early emergence could be favored by natural selection. However, as shown by McLachlan (1999), infestation by mite larvae can promote mating success in midges, and although McLachlan (1999) did not consider the survival rate of chironomid offspring, and used different species as model organisms, his findings would tend not to favor parasite regulated evolution of emergence period shifts in chironomids. On the other hand, if the pattern observed by McLachlan (1999) is, as suggested by Rolff (2001), caused by higher mating success of younger male midges, McLachland's data give an incorrect picture of mating success in infested midges. In computer simulations of the evolutionary relationship between the water mite Arrenurus cuspidator and its host the damselfly Coenagrion puella, Rolff et al. (2001) found that the parasites can exert severe selection pressure on the hosts if the damselflies are allowed to react by shifting their emergence period. However, field observations of this parasite-host relationship are more in accordance with the model where the parasite alone is allowed to evolve and exploit the whole emerging population of damselflies (Rolff et al. 2001). Thus, the damselfly emergence period apparently is constrained by factors more important than the effect of parasitism by the water mite. A similar scenario is not unlikely for spring chironomids and their water mite parasites, since many species have distinct and relatively short emergence periods. We believe that the answer to our above question is complex, and probably species specific since different species are subjected to different selection pressures and selection on different aspects of their biology.

\section{Conclusion and suggestions for further studies}

Chironomid species richness and the bias towards orthoclads observed in the seven Luxembourg springs were about as high as could be expected from previous 
studies on European cold water springs. The observed diversity of water mites was also as expected, but there are not many comparable studies available. Our data reveal a good deal of variation in terms of prevalence for Chironomidae species, and various reasons can be suggested to explain the observed variation, some of which provide testable hypotheses for future studies. Laboratory experiments to test host preference and selection for various spring-living water mites on their potential chironomid host is an attractive approach to suggest for future investigations. However, our experience is that spring living chironomids are difficult to culture in suboptimal conditions in the laboratory, thus this type of experimental set up needs perfection to be successful.

\section{Acknowledgements}

This study is part of the results of a project conducted by the research centre of the National Museum of Natural History of Luxembourg. We thank Isabel Schrankel for collecting the material and Marc Mayer and Reinhard Gerecke for initiating the faunistic survey of Luxembourg springs. Reinhard Gerecke is also thanked for valuable comments on the manuscript. Thanks are also due to Ole A. Sæther for aid in identifying difficult chironomid taxa, to Bjarne A. Meidell and Bergen Museum for generously offering research facilities to the senior author, and to anonymous reviewers for their comments and suggestions for improvements on the manuscript.

\section{References}

Booth J.P. \& Learner M.A. 1978. - The parasitization of chironomid midges (Diptera) by water-mite larvae (Hydracarina: Acari) in a eutrophic reservoire in South Wales. Arch. Hydrobiol., 84, 1-28.

Cantonati M. \& Ortler K. 1998. - Using spring biota of pristine mountain areas for long-term monitoring. IAHS Publ., 248, 379385.

Cranston P.S., Oliver D.R. \& Sæther O.A. 1989. - The adult males of Orthocladiinae (Diptera: Chironomidae) of the Holarctic region Keys and diagnoses. Pages 165-352 in Chironomidae of the Holarctic region. Keys and diagnoses. Part 3. Adult males. Wiederholm T. (eds). Ent. scand., Suppl., 34.

Efford I.E. 1963. - The parasitic ecology of some watermites. $J$. Anim. Ecol., 32, 141-156.

Ferrington L.C. 1998. - Generic composition of the chironomid fauna in springs of North America. Pages 141-155 in Studies in crenobiology. The biology of springs and springbrooks. Botosaneanu L. (eds). Backhuys Publishers, Leiden.

Fittkau E.J. 1962. - Die Tanypodinae (Diptera: Chironomidae) (Die Tribus Anatopyggiini, Macropelopiini und Pentaneurini). $A b$ handlungen zur Larvalsystematik der Insekten, 6, 1-453.

Gerecke R., Meisch C., Stoch F., Acri F. \& Franz H. 1998. - Eucrenon-hypocrenon ecotone and spring typology in the Alps of Berchtesgaden (upper Bavaria, Germany). A study of Microcrustacea (Crustacea : Copepoda, Ostracoda) and water mites (Acari Halacaridae, Hydrahnellae). Pages 167-182 in Studies in crenobiology. The biology of springs and springbrooks. Botosaneanu L. (eds). Backhuys Publishers, Leiden.

Gerecke R., Stoch F., Meisch C. \& Schrankel I. 2005. - Die Fauna der Quellen und des hyporheischen Interstitials in Luxemburg unter besonderer Berücksichtigung der Milben (Acari), Muschel- krebse (Ostracoda) und Ruderfusskrebse (Copepoda). Ferrantia, 41, 1-139

Gümbel D. 1976. - Emergenz-Vergleich zweier Mittelgebirgsquellen 1973. Schlitzer Produktionsbiologische Studien (18). Arch. Hydrobiol. Suppl., 50, 1-53.

Hevers J. 1978. - Interspezifische Beziehungen zwischen Unionicola-Larven (Hydrachnellae, Acari) und Chironomidae (Diptera, Insecta). Verh. Ges. Ökol. (1977). 7. Jahresversammlung, 211-217.

Kouwets F.A.C. \& Davids C. 1984. - The occurrence of chironomid imagines in an area near Utrecht (the Netherlands), and their relations to water mite larvae. Arch. Hydrobiol., 99, 296-317.

Lanciani C.A. \& Boyt A.D. 1977. - The effect of a parasitic water mite, Arrenurus pseudotenuicollis (Acari : Hydrachnellae), on the survival and and reproduction of the mosquito Anopheles crucians (Diptera: Culicidae). J. Med. Entomol., 14, 10-15.

Lehmann J. 1971. - Die Chironomiden der Fulda (Systematische, ökologische und faunistische Untersuchungen). Arch. Hydrobiol. Suppl., 37, 467-555.

Lindegaard C. 1995. - Chironomidae (Diptera) of European cold springs and factors influencing their distribution. J. Kansas Entomol. Soc., 68, 108-131.

Lindegaard C., Brodersen K.P., Wiberg-Larsen P. \& Skriver J. 1998. - Multivariate analyses of Macrofaunal communities in Danish springs and springbrooks. Pages 201-219 in Studies in crenobiology. The biology of springs and springbrooks. Botosaneanu L. (eds). Backhuys Publishers, Leiden.

Lindegaard C., Thorup J. \& Bahn M. 1975. - The invertebrate fauna of the moss carpet in the Danish spring Ravnkilde and its seasonal, vertical, and horizontal distribution. Arch. Hydrobiol., 75, 109-139.

Margolls L., Esch G.W., Holmes J.C., Kurls A.M. \& Schad G.A. 1982. - The use of ecological terms in parasitology (Report of an ad hoc committee of the American Society of Parasitologists). $J$. Parasitol., 68, 131-133.

Martin P. 2004. - Specifity of attachment sites of larval water mites (Hydrachnidia, Acari) on their insect hosts (Chironomidae diptera) - evidence from some stream-living species. Exp. Appl. Acarol., 34, 95-112.

Martin P. \& Otto C.-J. 2004. - Wassermilben (Hydrachnidia, Acari) als Parasiten von Insekten - Befunde von einem norddeutschen Tieflandbach. Deut. Gesell. Limnol. (DGL) - Tagungsbericht 2003 (Köln), Berlin 2004, 496-501.

Martin P. \& Stur E. 2005 - On the attachment sites of larval water mites (Acari, Hydrachnidia) from freshwater springs on their nematoceran hosts (Insecta, Diptera). Arch. Hydrobiol., 164, 89-107.

Martin P., Wiedenbrug S., Stur E. \& Schrankel I. 2002. - Wassermilben (Hydrachnidia, Acari) als Parasiten an quellbewohnenden Zuckmücken (Chironmidae, Diptera) - erste Befunde aus dem Nationalpark Berchtesgaden. Deut. Gesell. Limnol. (DGL) - Tagungsbericht (Kiel), 691-696.

McLachlan A. 1999. - Parasites promote mating success: the case of a midge and a mite. Anim. Behav., 57, 1199-1205.

McLachlan A., Ladle R. \& Bleay C. 1999. - Is infestation the result of adaptive choice behaviour by the parasite? A study of mites and midges. Anim. Behav., 58, 615-620.

Nolte U. 1991. - Seasonal dynamics of moss-dwelling chironomid communities. Hydrobiologia, 222, 197-211.

Orendt C. 2000. - The chironomid communities of woodland springs and spring brooks, severely endangered and impacted ecosystems in a lowland region of eastern Germany (Diptera: Chironomidae). J. Insect Cons., 4, 79-91.

Reiss F. 1984. - Neostempellina thienemanni n. gen., n. sp., eine europäische Chironomide mit gehäusetragenden Larven (Diptera, Insecta). Spixiana, 7, 203-210. 
Rolff J. 2001. - Evolutionary ecology of water mite-insect interactions: a critical appraisal. Arch. Hydrobiol., 152, 353-368.

Rolff J., Vogel C. \& Poethke, H-J. 2001. - Co-evolution between ectoparasites and their insect hosts: a simulation study of a damselfly-water mite interaction. Ecol. Entomol., 26, 638-645.

Sæther O.A. 1990. - A review of the genus Limnophyes Eaton from the Holarctic and Afrotropical regions (Diptera : Chironomidae, Orthocladiinae). Ent. scand. Suppl., 35, 139 p.

Smith B.P. 1988. - Host-parasite interaction and impact of larval water mites on insects. Annu. Rev. Entomol., 33, 487-507.

Smith B.P. \& McIver S.B. 1983. - Factors influencing host selection and successful parasitism of Aedes spp. mosquitoes by Arrenurus spp. mites. Can. J. Zool., 62, 1114-1120.

Smith I.M., Cook D.R. \& Smith B.P. 2001. - Water mites (Hydrachnida) and other arachnids. Pages 551-659 in Chapter 16 : Ecology and Classification of North American Freshwater Invertebrates. Thorp J.H. \& Covich A.P. (eds). Academic Press, San Diego.

Smith I.M. \& Oliver D.R. 1976. - The parasitic associations of larval water mites with imaginal aquatic insects, especially Chironomidae. Can. Entomol., 108, 1427-1442.

Smith I.M. \& Oliver D.R. 1986. - Review of parasitic associations of larval water mites (Acari, Parasitengona, Hydrachnida) with insect hosts. Can. Entomol., 118, 407-472.

Smukalla R. \& Meyer E. 1988. - Insect emergence from a shallow southern West-German lake, with special reference to the parasitic host-associated water mite larvae. Hydrobiologia, 169, 149 166.

Stechmann D.H. 1980. - Zum Wirtskreis syntopischer ArrenurusArten (Hydrachnellae, Acari) mit parasitische Entwicklung an Nematocera (Diptera). Z. Parasitenk., 62, 267-283.

Strenzke K. 1950. - Systematik, Morphologie und Ökologie der terrestrischen Chironomiden. Arch. Hydrobiol. Suppl., 18, 207-414.

Stur E. \& Ekrem T. in press. - A revision of West Palaearctic species of the Micropsectra atrofasciata species group (Diptera: Chironomidae). Zool. J. Linn. Soc.

Stur E. \& Sæther O.A. 2004. - A new hairy-winged Pseudorthocladius (Diptera : Chironomidae) from Luxemburg. Aquatic Insects, 26, 79-83.

Stur E. \& Wiedenbrug S. in press. - Chironomidae. Pages in Quellen im Nationalpark Berchtesgaden. Faunistisch-ökologische Untersuchungen und Perspektiven für die langfristige Umweltbeobachtung. Gerecke R. (eds). Nationalpark Berchtesgaden Forschungsbericht, Berchtesgaden.

Stur E. \& Wiedenbrug S. 2005. - Two new orthoclad species (Diptera: Chironomidae) from cold water springs of the Nationalpark Berchtesgaden, Germany. Aquatic Insects, 27, 125-131.

Thienemann A. 1954. - Chironomus. Leben, Verbreitung und wirtschaftliche Bedeutung der Chironomiden. Die Binnengewässer, 20, 834 p.

Ullrich F. 1978. - Biologisch-ökologische Studien an den Larven rheophiler Wassermilben (Hydrachnellae, Acari). Schlitzer Produktionsbiologische Studien. Arch. Hydrobiol. Suppl., 54, 189 255.

Wagensonner I. 1992. - Faunistische Untersuchung (Makroinvertebraten) des Herbst- und Winteraspektes eines Quelltümpels in der nördlichen Pupplingen Au, Oberbayern. Zoologisches Institut, Ludwig-Maximilians-Universität, München, 123 p.

Wiederholm T. 1989. - Chironomidae of the Holarctic region. Keys and diagnoses. Part 3. Adult males. Ent. scand., Suppl., 34, 532 p.

Wiles P.R. 1987. - Observation on the parasitic biology of the watermite Hydrodroma despiciens pisola Besseling (Acari: Hydrodromidae). Arch. Hydrobiol. Suppl., 76, 369-392.

Williams D.D. \& Williams N.E. 1998. - Invertebrate communities from freshwater springs: what can they contribute to pure and applied ecology? Pages 251-261 in Studies in crenobiology. Botosaneanu L. (eds). Backhuys, Leiden. 